\title{
Intensive Care Unit Preparedness During Pandemics and Other Biological Threats
}

Ryan C. Maves, MD*, Christina M. Jamros, Do ${ }^{1}$, Alfred G. Smith, DO ${ }^{1}$

\section{KEYWORDS}

- Pandemic • Influenza • Disaster preparedness

\section{KEY POINTS}

- In a globalized world where epidemics and pandemics are increasingly common, hospital preparation requires early planning.

- Infection prevention controls are critical during pandemics to reduce the risk to staff and other patients.

- In an emergency, ICU capacity may be increased by utilizing alternate hospital sites and non-ICU staff under the supervision of trained critical care personnel.

- Community engagement during a pandemic is important, to reduce alarm and also to ensure equitable distribution of limited resources.

\section{INTRODUCTION}

Outbreaks of infectious disease pose unique challenges for hospitals and intensive care units (ICUs). In the twentieth century, the polio pandemic led to the development of mechanical ventilation and was a major driver for the first units devoted to the care of patients with respiratory failure. The 2009 to 2010 H1N1 influenza pandemic, with

Disclosure: The authors have no financial conflicts of interest to disclose. The authors are US military service members. This work was prepared as part of their official duties. Title 17 U.S C. $\$ 105$ provides that "Copyright protection under this title is not available for any work of the United States Government." Title 17 U.S C. $\$ 101$ defines a US Government work as a work prepared by a military service member or employee of the US Government as part of that person's official duties. The views expressed in this article are those of the authors and do not necessarily reflect the official policy or position of the Departments of the Navy, the Department of Defense, nor the US Government.

Division of Infectious Diseases, Department of Internal Medicine, Naval Medical Center, 34800 Bob Wilson Drive, San Diego, CA 92134, USA

${ }^{1}$ These authors contributed equally to this article.

* Corresponding author.

E-mail address: ryan.c.maves.mil@mail.mil 
previously healthy victims suffering from severe respiratory failure and refractory hypoxemia, helped drive the expansion of extracorporeal membrane oxygenation (ECMO) from an infrequently used salvage therapy to a major critical care intervention. Outbreaks of severe acute respiratory syndrome-associated coronavirus (SARS-CoV) disease in 2001 to 2002 and the 2013 to 2014 West African Ebola epidemic showed the risks of highly contagious disease to clinical staff and the potential of infectious diseases to spread quickly between continents.

Although sometimes used interchangeably, the differences between the terms "outbreak," "epidemic," and "pandemic" are primarily ones of scale. Outbreaks are local increases in disease incidence that may place strain on a single hospital or several hospitals in a region. In industrialized countries, the impact of outbreaks on critical care resources may be limited because of the availability of patient transfer to other facilities. Epidemics are similar to outbreaks in that they refer to an often sudden increase in the rate of a disease in a geographic area, but the common usage of the word "epidemic" implies a larger geographic area than an outbreak, with a greater potential impact on health care resources. A pandemic is an epidemic that affects multiple areas of the world; in the case of influenza, a pandemic is formally defined by the World Health Organization (WHO) as an epidemic occurring in at least 2 different nations in 2 different $\mathrm{WHO}$ regions.

The potential infectious threats to a hospital and its associated ICUs are numerous and varied, including antimicrobial-resistant pathogens, nosocomial infections, and common and predictable dangers such as seasonal influenza. It is not hyperbole to describe carbapenem-resistant Enterobacteriaceae as a pandemic threat, for example. These sorts of infections, however, place gradual strains on hospital systems and only rarely cause acute disasters.

The sorts of infections most likely to require a disaster-level response for ICUs in industrialized settings are respiratory viruses, specifically influenza, although coronaviruses have produced similar sorts of outbreaks with SARS-CoV and the more recent Middle Eastern Respiratory syndrome-associated coronavirus (MERS$\mathrm{CoV}$ ). Limitations in public health infrastructure in resource-constrained regions, such as reliable, clean water and vector control, may lead to epidemics of virulent but less-contagious pathogens such as cholera; natural disasters may directly cause or exacerbate such epidemics, as in Haiti following the January 2010 earthquake.

Hemorrhagic fever viruses have led to epidemics with global pandemic potential, as dramatically demonstrated by Ebola and related viruses. The unique infection prevention requirements, the intensity of staffing, and sophisticated training required for the care of viral hemorrhagic fevers mean that a small number of patients can easily overwhelm a health system.

For purposes of planning, the types of infectious diseases that are most likely to be implicated in disaster share certain key characteristics:

\section{Virulence}

A significant proportion of infected patients need to be at risk for organ failure and death. This proportion may be a minority of affected patients, but the risk of severe disease must be substantial and may not necessarily impact groups who are at "typical" risk for critical illness (eg, the elderly and immunocompromised).

\section{Contagiousness}

There may be a high risk of infection following exposure to an ill patient. Alongside this risk of transmission, the population at risk of exposure should generally lack immunity 
to the infecting pathogen. If a given epidemic pathogen is not especially contagious (ie, cholera), then it may result from an exposure that is difficult to avoid, such as widespread contamination of drinking water.

Although history provides numerous examples of public health emergencies due to infection, the 2003 outbreak of SARS-CoV, the 2009 to 2010 influenza A(H1N1) pandemic, and the limited number of Western patients infected with Ebola virus in 2014 to 2015 serve as a useful basis for future hospital and ICU-based planning.

\section{Severe acute respiratory syndrome-associated coronavirus outbreaks, 2003}

In February 2003, 300 people in the Guangdong Province of China were reported as suffering from a novel and severe respiratory illness, with 5 confirmed deaths at the time. ${ }^{1,2}$ The following month, 11 health care workers (HCWs) were diagnosed with what was later named by the WHO as SARS-a coronavirus of presumably zoonotic origin. ${ }^{1,3,4}$ A physician from Guangdong, not previously known to be infected with SARS, subsequently traveled to Hong Kong, leading to infection of several other visitors to the hotel in which he was staying. ${ }^{3}$ These newly infected individuals then traveled via airplane, spreading the virus to Vietnam, Singapore, Ireland, Canada, and the United States.

The bulk of SARS cases occurred in eastern Asia, although 33 cases and 1 death were identified in Europe, 29 cases and no deaths in the United States, and 251 total cases with 43 deaths in Canada. Transmission and subsequent infection rates were high following exposure to patients who were infected with SARS, with up to $60 \%$ of exposed nurses in a Toronto hospital subsequently falling ill before the imposition of effective controls. ${ }^{5}$ Among hospitalized patients, $10 \%$ to $20 \%$ progressed to hypoxemic respiratory failure requiring mechanical ventilation. ${ }^{6,7}$ By July 2003 , the outbreak had peaked with a total of 8096 documented infections with 774 deaths (case-fatality rate $9.6 \%$ ). ${ }^{3,8}$

Throughout the outbreak, case identification and early management were limited by the nonspecific initial symptoms of SARS, with a respiratory viral prodrome progressing to fulminant disease that could quickly overwhelm a smaller community hospital with limited resources and staff. ${ }^{9}$ Many hospitals lacked contingency plans for surge capacity, patient transfer, or sufficient numbers of trained staff. In addition, initial government delays in notifying the general public about the potential pandemic spread of this novel virus prevented hospitals from implementing proper infection control procedures, contributing to a disproportionate number of HCWs becoming infected with SARS. Similarly, an early lack of infection prevention protocols led to many of the earlier patients being assigned to rooms alongside uninfected patients, further increasing the spread of disease. ${ }^{10}$ Although the primary outbreak peaked in May 2003 , delayed secondary outbreaks were reported into June and July, in part due to relaxed infection control practices later in the epidemic. ${ }^{11}$

\section{Influenza $A(H 1 N 1)$ pandemic, 2009 to 2010}

Critical care services were challenged worldwide during the novel 2009 influenza $A$ (H1N1) pandemic. The first cases emerged in the southwestern United States and Mexico in early 2009. ${ }^{12}$ By late April 2009, reported cases of $\mathrm{A}(\mathrm{H} 1 \mathrm{~N} 1)$ had spread across North America and worldwide. By August 2010, when the pandemic had run its course, influenza had caused approximately 300,000 deaths worldwide, of which 200,000 were due to respiratory failure. Unlike typical influenza seasons, higher mortality was documented among children, young adults, and pregnant women. ${ }^{13}$

In Mexico City alone, 58 of 899 patients (6.5\%) with influenza A(H1N1) hospitalized at 6 hospitals between March and June 2009 required critical care admission, a 
significant burden in facilities who averaged only 16 ICU beds per hospital. ${ }^{12}$ In that setting, over a strained hospital system and delays in admission because of ICU overcrowding, there was an overall 60 -day mortality of $41 \%$ of critically ill patients and an increased risk of death in critically ill patients receiving care outside of the ICU.

Even in higher-resource settings, ICU capacity was strained. Australia and New Zealand reported an 8-fold increase in influenza incidence during the southern hemispheric winter, compared with the same period in the United States. ${ }^{14}$ In a cohort study of all ICUs in Australia and New Zealand from June through August 2009, $5.2 \%$ of ICU bed-days were from patients with $2009 \mathrm{H} 1 \mathrm{~N} 1$ influenza and reached a peak percentage of ICU bed occupancy of $8.9 \%$ to $19.0 \%$. The most significant effect on ICU resource consumption occurred 4 to 6 weeks after the first confirmed winter ICU admission, with extra workload lasting several weeks. ${ }^{14}$

In Canada, a prospective observational study of 38 adult and pediatric ICUs evaluated 168 critically ill patients infected with $A(H 1 N 1)$, of whom 136 patients $(81.0 \%)$ required mechanical ventilation for a median duration of 12 days. Additional lung rescue therapies included neuromuscular blockade (28\% of patients), inhaled nitric oxide (13.7\%), high-frequency oscillatory ventilation (11.9\%), ECMO (4.2\%), and prone positioning ventilation (3.0\%). ${ }^{15}$ Ninety-day mortality in this cohort was $17.3 \%$, less than in Mexico City, but still substantial despite the increased resources that could be brought to bear.

\section{West Africa Ebola virus disease epidemic, 2014 to 2016}

In March 2014, the Ministries of Health in the West African nations of Guinea and Liberia reported outbreaks of Ebola virus disease (EVD) that rapidly spread to nearby countries and overseas. The resulting EVD epidemic affected over 28,000 persons in Guinea, Liberia, and Sierra Leone, in addition to smaller case numbers in Nigeria, Mali, and Senegal. Over 11,000 EVD victims died in the resulting outbreak in West Africa (WHO data).

A vastly smaller number of patients with EVD received treatment outside of Africa, including 11 in the United States and 16 in Europe. ${ }^{16}$ Of these 27 patients, the majority were infected in West Africa, but 2 patients were nurses who were secondarily infected following exposure to a man with EVD who had traveled recently to Liberia. These cases of imported EVD increased awareness of the risk of viral hemorrhagic fevers in a globalized world, but, more practically, it also demonstrated to clinicians in industrialized countries the intensity of resources, staffing, and training required to safely care for patients with EVD. The mortality of patients with EVD treated in industrialized countries was low compared with the experience in West Africa (5/27 total patients, or $18.5 \%),{ }^{17}$ with the successful implementation of critical care interventions previously thought to be futile, including mechanical ventilation and renal replacement therapy. At the same time, the low numbers of cases permitted an intensity of care in the United States and Europe that may not be feasible in a generalized outbreak.

Beyond the hospital, Ebola produced a unique public response. Widespread media reporting on the risks of EVD transmission led to public concern and occasional protests, ${ }^{18}$ as well as potential stigma and social isolation for international health workers returning home after caring for patients with EVD. ${ }^{19}$

\section{INTENSIVE CARE UNIT PLANNING FOR PANDEMICS}

As with any disaster impacting hospitals, the fundamental limitations of "stuff, staff, and space" apply to ICU planning for pandemics. Hospital staff can be and often are impacted personally by noninfectious disasters in their communities, such as hurricanes and earthquakes, with effects on their ability to come to work and care for patients. ${ }^{20,21}$ In caring for patients with infectious diseases during epidemics, however, 
much of the risk to HCWs is a direct result of their actual work and not a general risk to their community; as such, training and institutional precautions need to be implemented to both protect staff and reduce absenteeism. ${ }^{22,23}$

\section{"Stuff": Supply Requirements for Pandemic Preparedness}

In an epidemic or pandemic, supplies are at increased risk for being depleted rapidly. Per Joint Commission requirements, accredited hospitals in the United States are required to plan for 96 hours of autonomous function without resupply, although this does not imply "full functional capacity" but rather the ability to care for existing patients and staff. Despite this requirement, shortages of routine supplies well within this 96 -hour limit have been described in recent disasters. ${ }^{24}$

Disposable items, such as pharmaceuticals (and particularly antimicrobial drugs), may be rapidly exhausted. ICU-specific reusable devices, most notably mechanical ventilators, may similarly be in limited supply, especially in a respiratory disease outbreak. There are approximately 62,000 full-feature ventilators (20 ventilators per 100,000 residents) in the United States. An influenza pandemic with a $30 \%$ attack rate and a high case-fatality rate could lead to a doubling of ventilator demand. ${ }^{25,26}$ When additional supplies are not available, alternative methods to provide respiratory support may need to be considered, such as the use of anesthesia ventilators, high-flow nasal cannula oxygenation, and noninvasive positive pressure ventilation for selected patients. ${ }^{27,28}$

In light of a known epidemic or pandemic, local, state, and federal health authorities require coordination to assess known available quantities of available required agents, such as oseltamivir and peramivir for influenza, plus appropriate antimicrobial drugs for secondary bacterial infections. In addition, planning needs to include "general" drugs used in the management of critically ill patients, such as intravenous fluids, agents for rapid-sequence intubation, analgesics and sedatives for intubated patients, vasopressors, venous thromboembolism prophylaxis, and neuromuscular blockade agents for patients with severe hypoxemic respiratory failure.

Given ongoing issues with drug shortages in the United States and elsewhere for commonly used agents, planning must also account for the need to identify alternative agents in the event of significant limitations in supply. ${ }^{29}$ It is worth noting that not all alternative agents are equivalent in efficacy to preferred first-line therapies, as illustrated in recent US shortages of norepinephrine, ${ }^{30}$ although this may be considered acceptable in a pandemic setting.

Although resupply from outside of an affected geographic area may be practical during a localized epidemic, a major pandemic could easily lead to nationwide shortages of routine agents. The US Department of Health and Human Services maintains the Strategic National Stockpile, which includes a stockpile of emergency pharmaceuticals, intravenous fluids, mechanical ventilators, and investigational agents available for emergency use (eg, brincidofovir for a poxvirus outbreak). ${ }^{31}$

High-containment pathogens, such as Ebola have special supply requirements. Ventilators, laboratory equipment, and other routine medical devices need to be specifically dedicated to patients with EVD. ${ }^{32}$ Medical waste production for patients with EVD is exceptionally high, with a single patient's management generating enough waste to fill 8 drums $(60 \mathrm{~L})$ in 1 day in the Netherlands. ${ }^{33}$ Procedures for autoclaving solid waste and decontaminating liquid waste before disposal in municipal systems are mandatory. ${ }^{34}$

\section{"Staff": Increasing the Safety of Trained Personnel}

Preserving a hospital's critical care capability during pandemics depends on trained personnel. When faced with an overwhelming number of ill patients, capacity may 
be increased by having trained critical care personnel supervise staff experienced in acute care (eg, hospitalists, medical/surgical nurses, general inpatient pharmacists), intervening directly for highly complex patients as well as for emergencies and procedures. ${ }^{35}$

HCWs may themselves fall victim to a pandemic, either in the community or at the bedside. ${ }^{36}$ In addition to general principles of surge response described elsewhere in this issue, staff safety needs to be maintained through careful infection prevention practices, the use of personal protective equipment (PPE), and medical countermeasures, such as vaccines or chemoprophylaxis as appropriate.

For seasonal influenza outbreaks, droplet precautions (separate rooms without dedicated negative-pressure systems) have been considered adequate. During the H1N1 pandemic, conventional surgical masks seemed sufficient as PPE for HCWs when compared with N95 respirators, although there may have been an advantage to $\mathrm{N} 95$ masks for laboratory staff. ${ }^{37} \mathrm{HCW}$ compliance with influenza vaccination has been historically inadequate; strong encouragement from institutional leadership and consideration for mandatory vaccination may be recommended. ${ }^{38}$

Novel respiratory viruses, such as a novel SARS-like or MERS-like coronavirus, will require novel preventive strategies to maintain staff safety. It is prudent to place such patients into negative-pressure rooms with airborne and contact isolation, with staff wearing N95 respirators, gowns, gloves, and shoe covers until further guidance from national and international public health authorities can be obtained. Active surveillance of patients admitted with respiratory disease, relocation of emergency department triage to a unit outside of the physical hospital, and establishment of dedicated units for patients with confirmed disease are similarly advisable, as was done with the SARS epidemic. ${ }^{10}$ Aerosolizing procedures, such as endotracheal intubation, bronchoscopy, and tracheostomy, can be performed using purified air-powered respirators (PAPRs). ${ }^{10}$ PAPRs may also be an alternative to N95 masks when negativepressure isolation rooms are not available in sufficient numbers for affected patients, although formal training in the proper use of these devices is necessary in advance.

Ebola and other high-containment pathogens require higher levels of expertise and precaution than even a respiratory virus. Following intensive initial training, the care of patients with EVD requires regular refresher training, with dedicated space for patient and staff safety as well as waste management, laboratory diagnostics, and the like. Full contact precautions and the use of PAPRs are routine in most US Ebola treatment units (ETUs). Training for hospital staff is available through the National Ebola Training and Education Center (www.netec.org).

Although having an adequate number of trained personnel is critical during an epidemic, "presenteeism," the act of coming to work while ill, is a significant threat to staff safety. Surveys during previous influenza years reported that over $40 \%$ of HCWs might come to work while suffering from respiratory infections, increasing the risk of transmission to staff and to patients. ${ }^{39}$ Even in settings of potential staff shortages, institutions must maintain policies to prevent HCWs with symptoms of the disease to avoid work and to seek appropriate medical care for themselves.

\section{"Space": Critical Care Without an Intensive Care Unit}

Although the practice of critical care is generally linked to the ICU as a location, critically ill patients may need to receive care outside of a traditional ICU in disaster settings. Considerations of infection prevention and the avoidance of crosscontamination may additionally dictate that infected patients be placed in a geographically separate location. In situations where there are inadequate numbers of individual 


\section{Box 1}

Planning for pandemics

1. Triage and resource allocation. If a disease outbreak overwhelms local capacity, even at surge levels, institutions will need to determine a just allocation of scarce resources. If hospital transfer outside of the affected region is possible, this will require coordination by regional and national authorities. Scarce resources, such as ECMO or access to an ETU, will require allocation in a manner that is open, consistent, and based on broadly accepted ethical principles. These decisions will benefit from intensivist input but cannot be the sole decision of intensivists. Critical care admission may need to be limited to patients with reasonable chances of survival, along with the use of ventilators if in shortage. The states of New York ${ }^{43}$ and Maryland ${ }^{44}$ have published ventilator allocation guidelines that use community engagement to best reflect local priorities and values. In all cases, critically ill patients denied ICU admission in a disaster must receive appropriate and compassionate palliative care.

2. Optimization of staffing. For hospitals that maintain an ETU capability, regular refresher training must be exercised to preserve perishable skills. For all hospitals, appropriate staff training on infection prevention practices for pandemic threats are a key part of training; "just-in-time" training in the event of an outbreak may be needed to augment routine training, but a core group of staff trained in pandemic diseases, including donning and doffing of PPE and PAPR use, should be routine for institutions.

3. Equipment, supplies, and space. In addition to maintaining the Joint Commission-mandated 96-hour supply requirement (including food, water, consumables, and medications), hospitals should have plans for obtaining additional supplies in coordination with nearby hospitals, pharmaceutical vendors, and regional and national health authorities, including via the Strategic National Stockpile. PAPRs, negative-pressure rooms, and anticipated bed capacity in different surge levels should be defined in advance. These surge plans need to include plans for alternate care sites within the institution for ICU-level patients, such as PACUs, as noted above, as well as plans for transfer for patients when capabilities are ultimately overwhelmed. Lastly, hospitals must ensure proper staff vaccination and discourage "presenteeism" for ill personnel.

4. Public health. Hospitals and ICUs must have plans to coordinate with public health authorities for identification of cases, access to diagnostics, and tracking of potentially contagious individuals as part of outbreak investigations. Systematic data collection, either through government, academic, or combined networks, is similarly crucial to test interventions to end an epidemic.

5. Public affairs. Hospitals should be transparent in their interactions with communities, while also protecting the privacy and dignity of their patients. In addition to community involvement in ethical decision-making, such as ventilator allocation, media interactions need to be done with the oversight of hospital administration, experienced public affairs personnel, and ideally in collaboration with public health authorities.

rooms, patients with confirmed infections may be cohorted in common areas to reduce the risk of cross-contamination. ${ }^{40}$

During a standard hospital surge, it is anticipated that institutions can tolerate a $20 \%$ increase in critically ill patients with minimal impact. Increases of up to $200 \%$ of normal capacity may occur, but only at the cost of degraded capability and a possible modification in the standard of care. When ICU capacity is exceeded, less-ill patients may need to be transferred to other levels of care, such as patients not requiring mechanical ventilation, vasopressor support, or intensive neuromonitoring. Conversely, areas of the hospital capable of ICU-level monitoring, such as a postanesthesia care unit (PACU) or a monitored step-down unit, may need to be repurposed as temporary ICUs, especially if cohorting of seriously ill patients is planned. (Cancellation of elective 
surgical procedures may open up the PACU and same-day surgical units as auxiliary ICUs and step-down units, for example.)

Ebola and other high-containment pathogens require even-more specific dedicated space. As of 2016, there were 56 Centers for Disease Control and Preventiondesignated ETUs in the United States, not of all which have maintained their capabilities since the end of the West Africa epidemic. ${ }^{41}$ Space (and staff) allocation for the care of a single patient with EVD in a US ETU may require the loss of 6 or more "standard" beds in terms of capacity. ${ }^{41}$ Because the total number of designated ETU beds in the United States is less than 100, it would take relatively few patients with EVD or similarly contagious diseases to rapidly overwhelm current national capacity. ${ }^{42}$

\section{PLANNING FOR PANDEMICS}

The appropriate response to epidemics and pandemics, like any disaster, requires appropriate planning by ICUs and their associated hospitals. The precise response will necessarily vary based on the scale and severity of the pandemic (Box 1).

\section{SUMMARY}

Pandemics and epidemics are unique challenges for ICU preparedness. In a highly mobile, globalized world, infectious disease is no longer confined to fixed geographic regions. The risks of pandemic disease to clinical staff requires that institutions have mechanisms to protect their personnel while also providing adequate care to affected patients. Engagement of community partners is necessary to permit adequate data collection, to develop ethical standards for resource allocation, and to manage anxiety and expectations among the public.

\section{REFERENCES}

1. Hung LS. The SARS epidemic in Hong Kong: what lessons have we learned? J R Soc Med 2003;96:374-8.

2. Wong ATY, Chen H, Liu SH, et al. From SARS to avian influenza preparedness in Hong Kong. Clin Infect Dis 2017;64:S98-104.

3. Christian MD, Poutanen SM, Loutfy MR, et al. Severe acute respiratory syndrome. Clin Infect Dis 2004;38:1420-7.

4. Centers for Disease Control and Prevention. Update: outbreak of severe acute respiratory syndrome-worldwide, 2003. MMWR Morb Mortal Wkly Rep 2003;52: $241-6,8$.

5. Varia M, Wilson S, Sarwal S, et al. Investigation of a nosocomial outbreak of severe acute respiratory syndrome (SARS) in Toronto, Canada. CMAJ 2003;169: 285-92.

6. Lew TW, Kwek TK, Tai D, et al. Acute respiratory distress syndrome in critically ill patients with severe acute respiratory syndrome. JAMA 2003;290:374-80.

7. Booth CM, Matukas LM, Tomlinson GA, et al. Clinical features and short-term outcomes of 144 patients with SARS in the greater Toronto area. JAMA 2003;289: 2801-9.

8. Centers for Disease Control and Prevention (CDC). Severe acute respiratory syndrome (SARS) and coronavirus testing-United States, 2003. MMWR Morb Mortal Wkly Rep 2003;52:297-302.

9. Tsang KW, Ho PL, Ooi GC, et al. A cluster of cases of severe acute respiratory syndrome in Hong Kong. N Engl J Med 2003;348:1977-85. 
10. Cheng VC, Chan JF, To KK, et al. Clinical management and infection control of SARS: lessons learned. Antiviral Res 2013;100:407-19.

11. Ofner-Agostini M, Wallington T, Henry B, et al. Investigation of the second wave (phase 2) of severe acute respiratory syndrome (SARS) in Toronto, Canada. What happened? Can Commun Dis Rep 2008;34:1-11.

12. Dominguez-Cherit G, Lapinsky SE, Macias AE, et al. Critically ill patients with 2009 influenza A(H1N1) in Mexico. JAMA 2009;302:1880-7.

13. Dawood FS, Iuliano AD, Reed C, et al. Estimated global mortality associated with the first 12 months of 2009 pandemic influenza A H1N1 virus circulation: a modelling study. Lancet Infect Dis 2012;12:687-95.

14. Investigators Al, Webb SA, Pettila V, et al. Critical care services and 2009 H1N1 influenza in Australia and New Zealand. N Engl J Med 2009;361:1925-34.

15. Kumar A, Zarychanski R, Pinto R, et al. Critically ill patients with 2009 influenza A(H1N1) infection in Canada. JAMA 2009;302:1872-9.

16. Leligdowicz A, Fischer WA 2nd, Uyeki TM, et al. Ebola virus disease and critical illness. Crit Care 2016;20:217.

17. Uyeki TM, Mehta AK, Davey RT Jr, et al. Clinical management of Ebola virus disease in the United States and Europe. N Engl J Med 2016;374:636-46.

18. Sell TK, Boddie C, McGinty EE, et al. Media messages and perception of risk for Ebola virus infection, United States. Emerg Infect Dis 2017;23:108-11.

19. Gee S, Skovdal M. Public discourses of Ebola contagion and courtesy stigma: the real risk to international health care workers returning home from the West Africa Ebola outbreak? Qual Health Res 2018;28:1499-508.

20. Morris AM, Ricci KA, Griffin AR, et al. Personal and professional challenges confronted by hospital staff following hurricane sandy: a qualitative assessment of management perspectives. BMC Emerg Med 2016;16:18.

21. Ochi S, Tsubokura M, Kato S, et al. Hospital staff shortage after the 2011 triple disaster in Fukushima, Japan - an earthquake, tsunamis, and nuclear power plant accident: a case of the Soso district. PLoS One 2016;11:e0164952.

22. Shiao JS, Koh D, Lo LH, et al. Factors predicting nurses' consideration of leaving their job during the SARS outbreak. Nurs Ethics 2007;14:5-17.

23. Maunder RG, Lancee WJ, Balderson KE, et al. Long-term psychological and occupational effects of providing hospital healthcare during SARS outbreak. Emerg Infect Dis 2006;12:1924-32.

24. Abramson DM, Redlener I. Hurricane sandy: lessons learned, again. Disaster Med Public Health Prep 2012;6:328-9.

25. Meltzer MI, Patel A, Ajao A, et al. Estimates of the demand for mechanical ventilation in the United States during an influenza pandemic. Clin Infect Dis 2015; 60(Suppl 1):S52-7.

26. Rubinson L, Vaughn F, Nelson S, et al. Mechanical ventilators in US acute care hospitals. Disaster Med Public Health Prep 2010;4:199-206.

27. Rubinson L, Branson RD, Pesik N, et al. Positive-pressure ventilation equipment for mass casualty respiratory failure. Biosecur Bioterror 2006;4:183-94.

28. Papazian L, Corley A, Hess D, et al. Use of high-flow nasal cannula oxygenation in ICU adults: a narrative review. Intensive Care Med 2016;42:1336-49.

29. Mazer-Amirshahi M, Goyal M, Umar SA, et al. U.S. drug shortages for medications used in adult critical care (2001-2016). J Crit Care 2017;41:283-8.

30. Vail E, Gershengorn HB, Hua M, et al. Association between US norepinephrine shortage and mortality among patients with septic shock. JAMA 2017;317: 1433-42. 
31. Redd SC, Frieden TR. CDC's evolving approach to emergency response. Health Secur 2017;15:41-52.

32. Garibaldi BT, Chertow DS. High-containment pathogen preparation in the intensive care unit. Infect Dis Clin North Am 2017;31:561-76.

33. Haverkort JJ, Minderhoud AL, Wind JD, et al. Hospital preparations for viral hemorrhagic fever patients and experience gained from admission of an Ebola patient. Emerg Infect Dis 2016;22:184-91.

34. Le AB, Hoboy S, Germain A, et al. A pilot survey of the U.S. medical waste industry to determine training needs for safely handling highly infectious waste. Am J Infect Control 2018;46:133-8.

35. Einav S, Hick JL, Hanfling D, et al. Surge capacity logistics: care of the critically ill and injured during pandemics and disasters: CHEST consensus statement. Chest 2014;146:e17S-43S.

36. Suwantarat N, Apisarnthanarak A. Risks to healthcare workers with emerging diseases: Iessons from MERS-CoV, Ebola, SARS, and avian flu. Curr Opin Infect Dis 2015;28:349-61.

37. Smith JD, MacDougall CC, Johnstone J, et al. Effectiveness of N95 respirators versus surgical masks in protecting health care workers from acute respiratory infection: a systematic review and meta-analysis. CMAJ 2016;188:567-74.

38. Bellia C, Setbon M, Zylberman P, et al. Healthcare worker compliance with seasonal and pandemic influenza vaccination. Influenza Other Respir Viruses 2013;7(Suppl 2):97-104.

39. Chiu S, Black CL, Yue X, et al. Working with influenza-like illness: presenteeism among US health care personnel during the 2014-2015 influenza season. Am J Infect Control 2017;45:1254-8.

40. Prevention strategies for seasonal influenza in healthcare settings. Available at: https://www.colc.gov/flu/professionals/infectioncontrol/healthcaresettings.htm. Accessed March 02, 2019.

41. Herstein JJ, Biddinger PD, Gibbs SG, et al. Sustainability of high-level isolation capabilities among US Ebola treatment centers. Emerg Infect Dis 2017;23:965-7.

42. Herstein JJ, Biddinger PD, Kraft CS, et al. Current capabilities and capacity of Ebola treatment centers in the United States. Infect Control Hosp Epidemiol 2016;37:313-8.

43. Powell T, Christ KC, Birkhead GS. Allocation of ventilators in a public health disaster. Disaster Med Public Health Prep 2008;2:20-6.

44. Daugherty Biddison EL, Faden R, Gwon HS, et al. Too many patients...A framework to guide statewide allocation of scarce mechanical ventilation during disasters. Chest 2019;155(4):848-54. 